\title{
Stability Study Pause Description Terminology
}

National Cancer Institute

\section{Source}

National Cancer Institute. Stability Study Pause Description Terminology. NCI Thesaurus.

Code C96083.

Terminology developed to support Pause Descriptions within the Stability Data

Standards. 